\title{
Design of Launch Vehicle Flight Control Systems Using Ascent Vehicle Stability Analysis Tool
}

\author{
Jiann-Woei Jang ${ }^{1}$, Abran Alaniz ${ }^{2}$, Robert Hall ${ }^{3}$ and Nazareth Bedrossian ${ }^{4}$ \\ The Charles Stark Draper Laboratory, Inc., Houston, TX, 77058 \\ and \\ Charles Hall ${ }^{5}$, and Mark Jackson ${ }^{6}$ \\ NASA Marshall Space Flight Center, Huntsville, AL, 35812
}

\begin{abstract}
A launch vehicle represents a complicated flex-body structural environment for flight control system design. The Ascent-vehicle Stability Analysis Tool (ASAT) is developed to address the complicity in design and analysis of a launch vehicle. The design objective for the flight control system of a launch vehicle is to best follow guidance commands while robustly maintaining system stability. A constrained optimization approach takes the advantage of modern computational control techniques to simultaneously design multiple control systems in compliance with required design specs. "Tower Clearance" and "Load Relief” designs have been achieved for liftoff and max dynamic pressure flight regions, respectively, in the presence of large wind disturbances. The robustness of the flight control system designs has been verified in the frequency domain Monte Carlo analysis using ASAT.
\end{abstract}

\section{Nomenclature}

$\phi_{C}=$ platform command angle $(\mathrm{rad})$

$\dot{\phi}_{C}=$ platform command rate $(\mathrm{rad} / \mathrm{sec})$

$\phi_{P}=$ platform angle (rad)

$\phi_{c . g .}=$ vehicle pitch angle about the center of gravity (rad)

$\alpha=$ angle of attack (rad)

$\beta_{E}=$ engine gimbal angle (rad)

$\beta_{C}=$ engine gimbal command angle (rad)

$G_{G D}=$ engine dynamics transfer function from gimbal command to gimbal angle (unitless)

$Z_{c . g}=$ displacement of vehicle c.g. normal to reference (ft)

$Z_{s j}=$ sloshing fluid displacement of $j^{\text {th }} \operatorname{tank}(\mathrm{ft})$

$m_{s j}=$ slosh mass in $j^{\text {th }}$ tank (slug)

$l_{s j}=$ c.g. to the $j^{\text {th }}$ slosh-mass distance (ft)

$M=$ total vehicle mass (slug)

$c_{1}=c_{z \alpha} q A\left(X_{\text {c.g. }}-X_{\text {c.p. }}\right) / I_{Z Z}$, aerodynamic acceleration coefficient $\left(1 / \mathrm{sec}^{2}\right)$

$c_{2}=F X_{\text {c.g. }} / I_{Z Z}$, vehicle angular acceleration $\left(1 / \mathrm{sec}^{2}\right)$

$I_{Z Z}=$ yaw vehicle moment of inertia $\left(\right.$ slug- $\left.\mathrm{ft}^{2}\right)$

$X_{\text {c.g. }}=$ center of gravity measured from the gimbal (ft)

$X_{c . p .}=$ center of pressure measured from the gimbal (ft)

$S_{E}=$ engine $1^{\text {st }}$ moment about gimbal (slug- $\mathrm{ft}$ )

$I_{E}=$ engine $2^{\text {nd }}$ moment about gimbal (slug- $\mathrm{ft}^{2}$ )

\footnotetext{
${ }^{1}$ Principal Member of the Technical Staff; Draper Laboratory, Houston TX, 77058: jang@draper.com, AIAA Senior Member ${ }^{2}$ Member of the Technical Staff; Draper Laboratory, Houston TX, 77058: aalaniz@draper.com

${ }^{3}$ Principal Member of the Technical Staff; Draper Laboratory, Houston TX, 77058: Robert.A.Hall@nasa.gov

${ }^{4}$ Group Leader, Manned Space Systems; Draper Laboratory, Houston TX, 77058: naz@draper.com

${ }^{5}$ Senior Aerospace Engineer; NASA Marshall Space Flight Center, Huntsville, AL 35812:Charles.E.Hall@nasa.gov

${ }^{6}$ Senior Aerospace Engineer; NASA Marshall Space Flight Center, Huntsville, AL 35812:Mark.E.Jackson@nasa.gov
} 


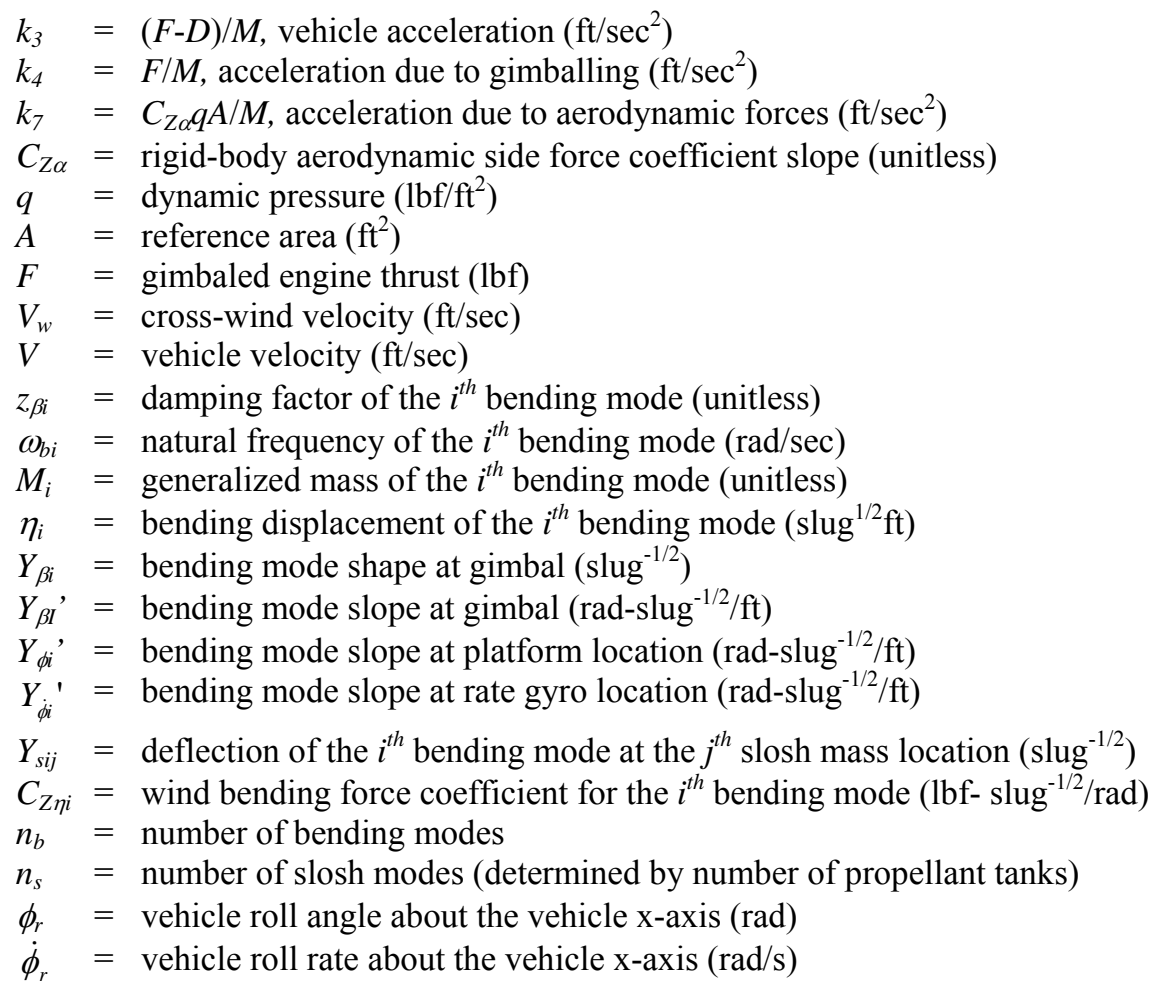

\section{Introduction}

A Launch Vehicle in general is referred to a rocket carrying some payload into outer space. There are several common characteristics which must be taken into account in designing the flight control system of a launch vehicle. First, a launch vehicle typically is aerodynamically unstable because the vehicle's center of pressure is above its center of gravity for most, if not all of its flight through the atmosphere. Second, the vehicle's mass, moment of inertia, aerodynamics, slosh, and bending characteristics vary greatly throughout the launch timeline. Third, low structural mass is desired but can be the source of significant flexible body dynamics as shown in Figure 1. Large flex response in the feedback loop may cause control structure interaction and eventually result in instability if excessive control gain and incorrect phasing are present ${ }^{1}$.

Ascent through the dense atmosphere is the most challenging phase in the entire flight. Launch tower clearance and dynamic load reduction are the most important tasks during atmospheric ascent. The aerodynamic load increases significantly right after liftoff, reaches its peak at the maximum dynamic pressure region and decreases greatly afterwards; the aerodynamic effect after the first stage flight is minimal. In order to alleviate the aerodynamic load during the first stage flight, a blended attitude control and load relief control scheme is designed to cancel out the unexpected large wind disturbance ${ }^{2,3}$. The primary goal of the attitude controller design is to ensure stable response to the guidance commands while minimizing trajectory deviations. However, the load relief controller will turn the vehicle in a weather-cocking fashion to reduce the aerodynamic load on the vehicle ${ }^{4}$. The competing design goals between attitude controller and load relief controller fall into the non-cooperation game category. The Pareto optimization method is used in this paper to generate a design that is a compromise between these competing goals in order to minimize the disturbance impact while closely tracking the reference trajectory ${ }^{1}$. The load relief controller for a launch vehicle can also be used to balance other types of disturbances due to hardware imperfections or build tolerances resulting in biased thrust vectors, thrust vector uncertainty and misaligned sensors.

Most launch vehicles use a Thrust Vector Control (TVC) system to gimbal the engine cone according to the output of the flight control system to minimize both pitch and yaw attitude errors. For a single gimbaled engine launch vehicle like Ares I, the roll attitude is controlled by a phase plane algorithm using fixed thruster firings. Some launch vehicles like Saturn V or Space Shuttle ${ }^{4}$ possess multiple gimbaled engines; roll attitude control is accommodated by differential thrust vectoring. For a launch vehicle with multiple gimbaled engines, asymmetric geometry and non-center line gimbaled engines will increase the coupling effect in the vehicle dynamics. 


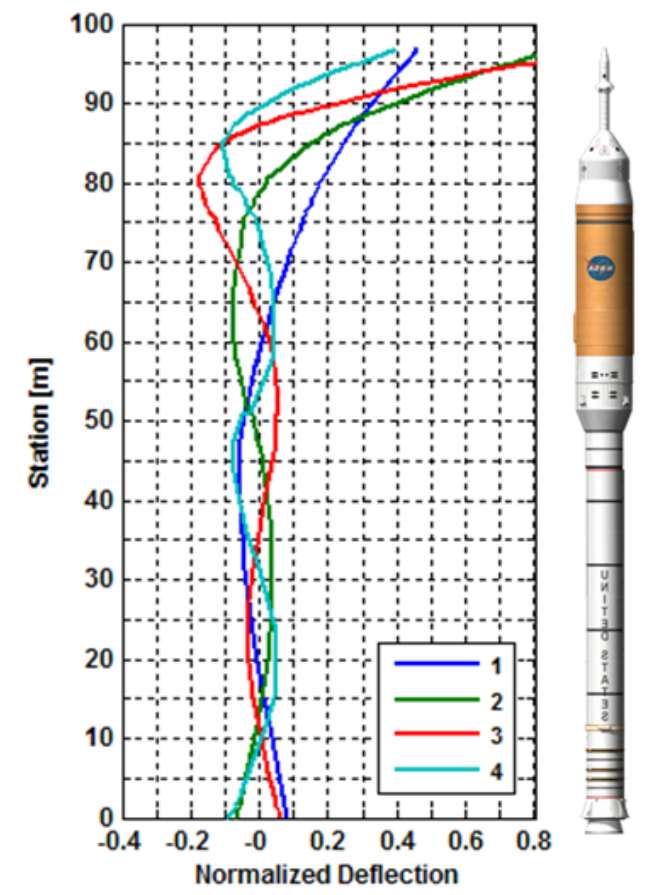

Figure 1. Modal displacements for Ares I launch vehicle

To increase the payload capacity, most launch vehicles are designed with low structural mass which results in a significantly flexible body. The frequency spectrum of a flight control system for a typical launch vehicle is shown in Figure 2. Bending frequencies are much lower than torsional frequencies. Flex filters in the flight control system are designed to minimize the flex components in the error signals while maximize the system performance. Notice that the first bending frequency interacts with the fuel slosh at some frequencies. The flex-slosh interaction must be taken into consideration in the flight control system design using bending filters ${ }^{1}$. 


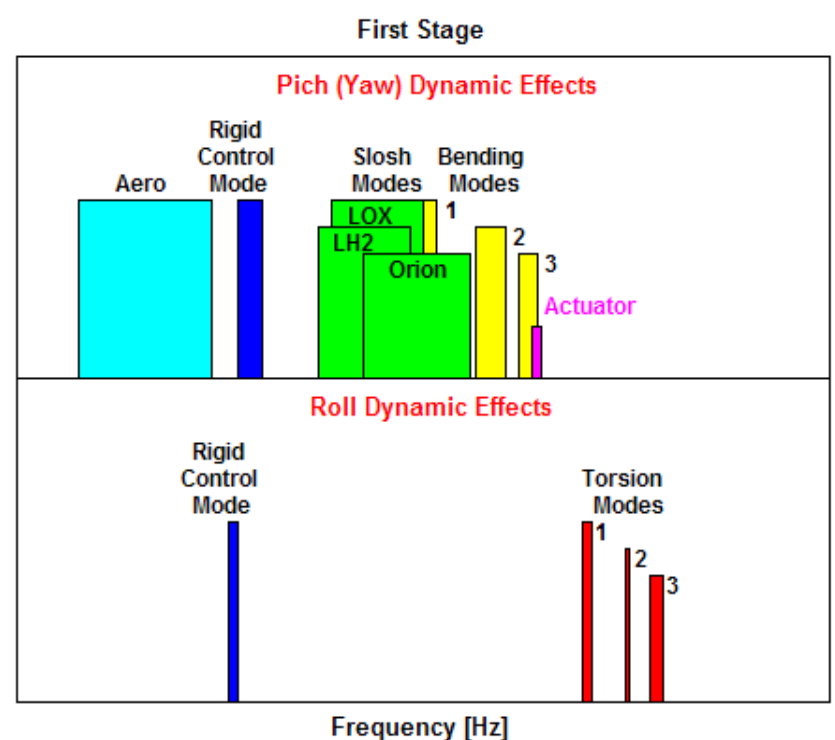

Figure 2. Ares-I First Stage Frequency Spectrum

Digital filter designs and application have been extensively investigated in the literature ${ }^{5-7}$. The most popular analog filter design approaches include Butterworth, Chebyshev and elliptic filters. Direct digital filter design methodologies are also available in the literature ${ }^{6}$. These methodologies have been used for open-loop system designs by shaping filters to meet the open-loop performance specifications in the frequency domain. Unfortunately, most of the closed-loop system stability/performance requirements cannot be directly mapped into open-loop system specifications. Recently, a robust controller design methodology was proposed using a numerical constrained optimization approach to maximize stability margins while meeting performance requirements ${ }^{8-13}$. This control design methodology has been successfully used to design a single robust control moment gyro (CMG) flex filter set for multiple International Space Station (ISS) stages $^{8}$ and robust flex filters and a PID controller for the Orbiter Repair Maneuver operation ${ }^{11}$.

The traditional flight control system design strategy using the "man-in-the-loop" and "one-system-design-at-atime" approach is not suited for flight system control design with competing design objectives such as trajectory tracking accuracy versus aerodynamic load alleviation or robust stability versus robust performance. To address the competing design goals, a methodology for employing numerical optimization is developed to recast such a flight control design problem as a constrained multi-objective optimization problem ${ }^{1}$. The design space includes the bending filters, PID gains and load relief gains and filters.

In this paper, a similar procedure used for the Ares I flight control system design ${ }^{1}$ is used to illustrate the capabilities of an ascent vehicle stability analysis tool. The design task can be divided into four phases to achieve desired performance and guaranteed robust stability:

(1) Optimal rigid controller designs which include an attitude controller for better trajectory tracking for the entire flight combined with an anti-drift/load relief controller to ensure tower clearance during liftoff and minimal aerodynamic load during the maximum aerodynamic pressure (Max Q) region.

(2) Sensor blending selection to robustly mitigate flex components from the sensed signals in the presence of flex uncertainty ${ }^{1}$.

(3) Flex filter designs to guarantee robust stability and adequate performance.

(4) High fidelity nonlinear simulation to verify stability and performance of control systems for entire flight.

These four design phases are not necessarily performed in sequence. For example, flex filter and controller designs can be combined into iterative design phases. In each of the first three phases, the design task is recast as a constrained optimization problem, which can be solved with modern computational optimization techniques. One advantage of this approach is that multiple flight control systems at multiple flight times can be designed simultaneously since the corresponding design constraints and objectives can all be included in the constrained 
optimization problem. Therefore, this approach will significantly reduce the cost and time for developing the flight control system of a launch vehicle.

To ease the complication of a launch vehicle flight control system design task, an effective design tool with a great deal of analysis capabilities within a user friendly environment is needed. The design and analysis tool should assess several common system configurations at any point in the launch timeline and perform robustness testing while maintaining an intuitive structure that can be easily navigated. It should also automatically generate results that are easy to interpret. One way to develop such a tool is by taking advantage of the pre-existing commercially available simulation development and analysis infrastructure. Known as commercial off the shelf (COTS) systems, these simulation and analysis environments are becoming the standard platforms for such applications. The desirable features of such an approach include ease of use, design modularity to provide dynamic scaling, and builtin analytical functions. Based on those criteria, Ascent-vehicle Stability Analysis Tool (ASAT) has been developed by Draper to satisfy these requirements by providing the system designer with a tool that is both powerful and easy to use. ASAT was developed using Matlab/Simulink software as the COTS platform and features a graphical user interface (GUI) through which users can select the dynamics model, flex filter, controller, and sensor architecture. ASAT can be easily used for flex filter, attitude and load relief controller design, sensor placement and blending studies, and stability analysis.

In this paper, launch vehicle dynamics and a flight control system architecture modeled in ASAT will be presented. The procedure to design the optimal sensor blending and recast the flight control design task into a constrained optimization problem will be introduced. A flight control system design example for a launch vehicle design will be provided. The robustness of the designed flight control system will be verified using ASAT MonteCarlo analysis.

\section{Flight Control System for Launch Vehicles}

In this section, the dynamics models used in ASAT will be briefly outlined. Notice that the cross coupling effect for a launch vehicle will be assumed small in the design phase. This allows the design task to be completed in each axis separately. This assumption, however, does not need to be used in the analysis phase; 6-DOF dynamics models are used in ASAT to verify the robustness the flight control system design.

\section{$\underline{\text { Pitch/Yaw Control System }}$}

The 2 DOF equations presented in their entirety in Reference 14-17 can be divided into rigid body dynamics, flex (bending) dynamics, and slosh dynamics. Figure 3 shows the sign conventions for the equations of motion with variables as defined in the List of Variables ${ }^{15}$. Note that the 2 DOF in this depiction include translation in the Y-axis and rotation in the Yaw. Linearized rigid body dynamics include rigid rotation about the center of gravity and the rigid body translation of the center of gravity. Specifically,

$$
\phi_{c . g .} s^{2}=-c_{1} \alpha-c_{2} \beta_{E}-\frac{1}{I_{z z}}\left(X_{c . g .} S_{E}+I_{E}\right) \beta_{E} s^{2}+\frac{1}{I_{z z}} \sum_{j=1}^{n_{s}} m_{s j}\left(l_{s j} s^{2}+k_{3}\right) Z_{s j}
$$

and

$$
Z_{c . g .} s^{2}=k_{7} \alpha+k_{4} \beta_{E}+k_{3} \phi_{c . g .}+\frac{S_{E}}{M} \beta_{E} s^{2}-\frac{1}{M} \sum_{j=1}^{n_{5}} m_{s j} Z_{s j} s^{2}
$$

Rigid body motion depends on forces and torques created by aerodynamic pressure, gimbal angle (including the "tail-wags-dog" effect), thrust, drag, and sloshing fuel masses. Angle of attack for the undeformed vehicle is a function of wind velocity $\left(V_{w}\right)$, rigid attitude $\left(\phi_{c . g .}\right)$, and rigid velocity $\left(Z_{c . g . s}\right)$

$$
\alpha=\left(V_{w} / V\right)+\phi_{c . g .}-\left(Z_{c . g .} s / V\right)
$$

Bending motion depends on flexibility characteristics such as natural frequency, damping, and generalized mass in addition to the forces mentioned above for the rigid body dynamics. Specifically, 


$$
\left(s^{2}+2 \xi_{b i} \omega_{b i} s+\omega_{b i}^{2}\right) M_{i} \eta_{i}=F Y_{\beta i} \beta_{E}+\left(S_{E} Y_{\beta i}-I_{E} Y_{\beta i}^{\prime}\right) \beta_{E} s^{2}-\sum_{j=1}^{n_{s}} m_{s j} Y_{s i j} Z_{s j} s^{2}+C_{Z \eta_{i}} \alpha
$$

Each bending mode is represented by a separate equation. The inclusion of more bending modes in a model leads to an increase in model fidelity. However, this also leads to an increase in the size of the system. The number of modes included in an analysis ultimately depends on the required model fidelity, the vehicle's frequency spectrum, and the available computation tools. For this reason, a modal selection algorithm is employed to include only the modes that significantly affect the control system design and attitude stability.

The dynamics of sloshing fuel is described by an equation for each fuel tank being considered in the analysis. Specifically,

$$
\left(s^{2}+2 \xi_{s j} \omega_{s j} s+\omega_{s j}^{2}\right) Z_{s j}=-Z_{c . g .} s^{2}+\left(l_{s j} s^{2}+k_{3}\right) \phi_{c . g .}-\sum_{i=1}^{n_{b}} Y_{s i j} \eta_{i} s^{2}
$$

Similar to bending modes, the inclusion of more slosh masses (up to the true number) yields a higher fidelity model. As shown in Equations 1, 2, and 4, the slosh tanks with greater slosh masses have a larger effect on overall vehicle motion. Equations 1 through 5 describe the overall yaw motion of the spacecraft and collectively form the plant dynamics.

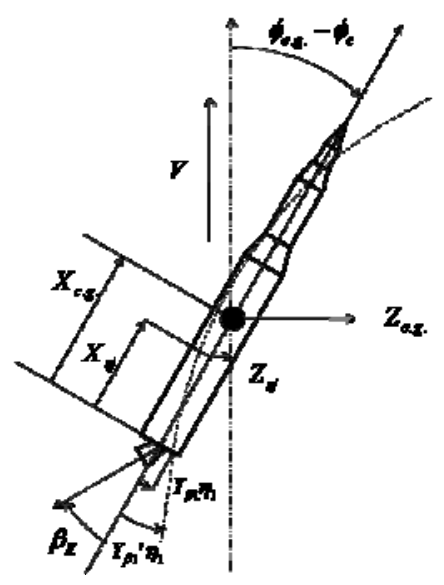

Figure 3. Spacecraft Sign Conventions

In addition to the plant dynamics, the control system includes a combination of other systems designed to regulate the spacecraft's attitude. Sensors on the body of the vehicle determine the attitude and attitude rate according to the equations

$$
\phi_{P}=\phi_{c . g .}+\sum_{i=1}^{n_{b}} Y_{\phi i}^{\prime} \eta_{i}
$$

and

$$
\phi_{R G} S=\phi_{c . g .} S+\sum_{i=1}^{n_{b}} Y_{\phi i}^{\prime} \eta_{i} S
$$

respectively. Note that, when convenient, the sensor equations can be included as part of the plant's output equations. Sensor output is filtered in order to attenuate the higher frequency bending modes ${ }^{14-17}$. The filter output becomes the controller input. The controller is a proportional-integral-derivative (PID) controller that outputs a gimbal command angle

$$
\beta_{C}=K_{I} \int\left(\phi_{P}-\phi_{C}\right)+K_{P}\left(\phi_{P}-\phi_{C}\right)+K_{D}\left(\dot{\phi}_{R G}-\dot{\phi}_{C}\right)
$$

The controller gains $\left(K_{I}, K_{P}\right.$, and $\left.K_{D}\right)$ are designed using gain scheduling, a process that involves optimizing a controller for specific design points throughout the time of flight and linearly interpolating between these points to 
obtain gain values for any flight time ${ }^{1,15}$. The command angle translates the gimbal angle via the dynamics of the actuator $\left(G_{G D}\right)$

$$
\beta_{E}=G_{G D} \beta_{C}
$$

The pitch and yaw attitude control system model as shown in Figure 4 includes the launch vehicle dynamics and attitude controller modules. The launch vehicle dynamics modules consist of both rigid and flex dynamics models ${ }^{1}$. The dynamics modules take in actual gimbal angles and output attitude and rate gyro measurements. The blended attitude and rate error signals represent the difference between commanded and actual attitude and rate. They are filtered by the attitude and rate filters, respectively. The filtered error signals are sent to the PID controller as shown in Figure 4 to generate the commanded gimbal angles, which drive the gimbal dynamics. The rate blending option can be used to actively reduce the effect of structural flexibility and the attitude blending option can be used to adjust vehicle rigid body performance.

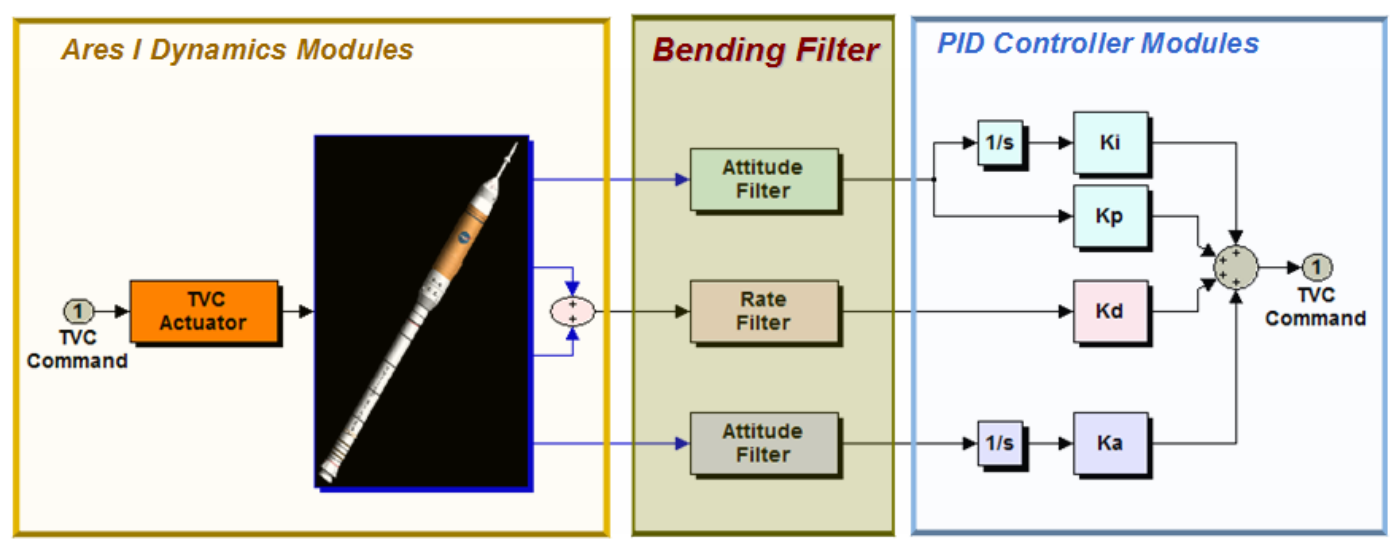

Figure 4. Launch Vehicle Attitude Control System Model

During first stage flight, a launch vehicle experiences large aerodynamic disturbances especially during max dynamic pressure flight regions. TVC gimbaled misalignment or sensor misalignment also result in undesired disturbances acting on the vehicle. To mitigate those disturbances, an anti-drift/load-relief controller is added in parallel to the basic attitude control architecture ${ }^{2}$ as shown in Figure 5. The anti-drift / load-relief algorithm includes force and moment balance feedbacks using input data from rate gyros and accelerometers. Signals from the anti-drift algorithm are added to the attitude control block to help alleviate vehicle loading, trajectory dispersions, thrust vector uncertainty, and center of gravity (CG) offsets. Two outputs come from the anti-drift / load-relief block - a force bias term, and a moment bias term. The force bias term is used to align the thrust vector in a direction to balance external forces, and the moment bias term is an input used to balance external moments. The actual implementation includes low-pass filtering to keep noise from causing undesired results, and to avoid control signal coupling with structural vibrations. Additionally, since the accelerometer data is from the IMU, which may have a radial offset from the centerline of the vehicle and not at the axial CG location, corrections to the acceleration signals are made due to body angular rates. The blended attitude control and anti-drift / load relief control laws require scheduled gains that are related to thrust, inertia, CG location, and weight. 


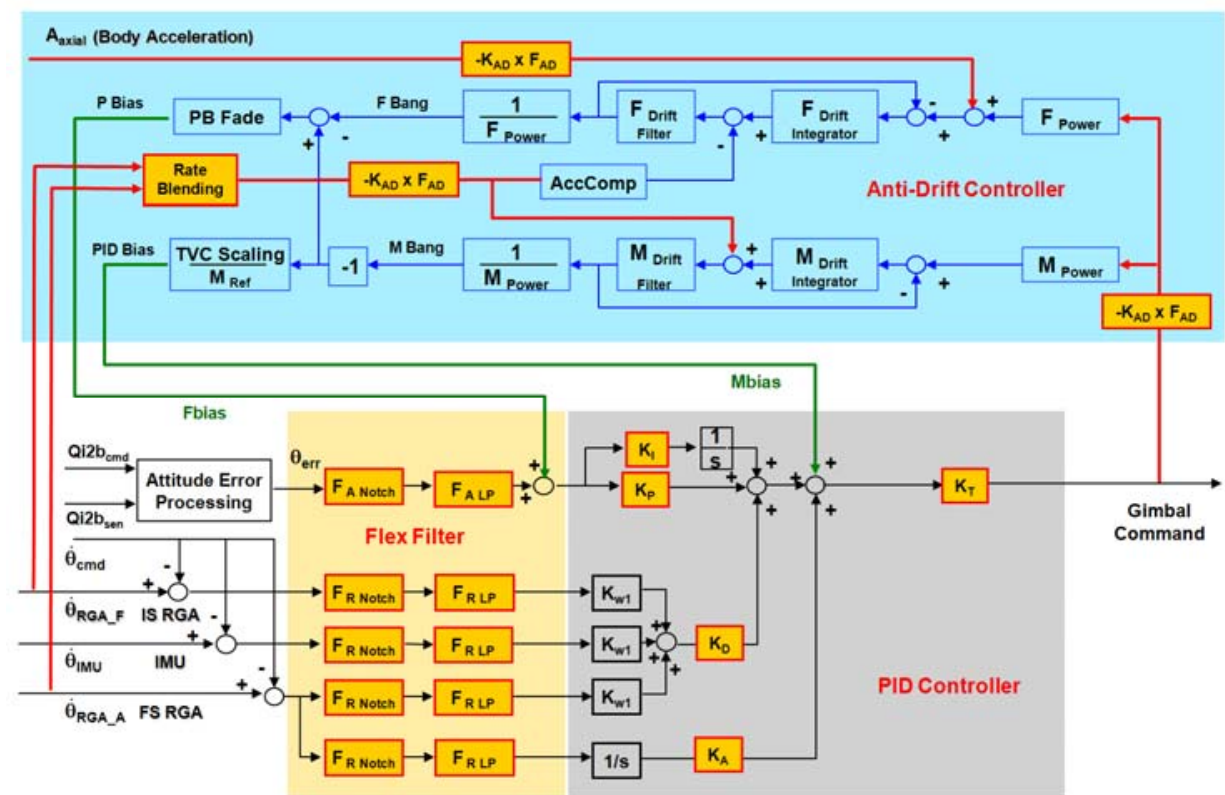

Figure 5. Ares-I Attitude and Load Relief / Anti-Drift Control System Model

One example of using the blended attitude and anti-drift control scheme is the liftoff clearance problem. In Figure 6, the yellow region represents an artificial keep-out zone to avoid the plume impingement on the launch tower (red region). In this example, a large gust comes from the left (South) of the launch tower. In Figure 6a, attitude controller by itself can guide the launch vehicle (blue lines) to clear the launch tower. However, when a TVC gimbaled bias is introduced as shown in Figure 6b, the resultant of gimbaled bias and gust disturbance is too large for the attitude controller to compensate, thus the launch vehicle flies into the tower. Without trying to cancel the undesired disturbance using the anti-drift controller, a fly away guidance is designed to maneuver the launch vehicle away from the tower. As shown in Figure 6c, the launch vehicle still is in the keep out zone with fly-away guidance only. Finally, in Figure 6d, the blended attitude/anti-drift control scheme with fly-away guidance turns the vehicle into the wind and successfully guides it through launch tower clearance. 


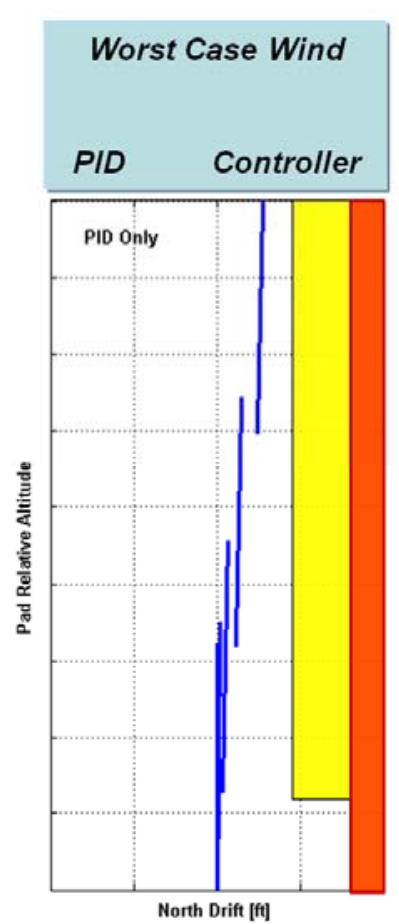

(a)

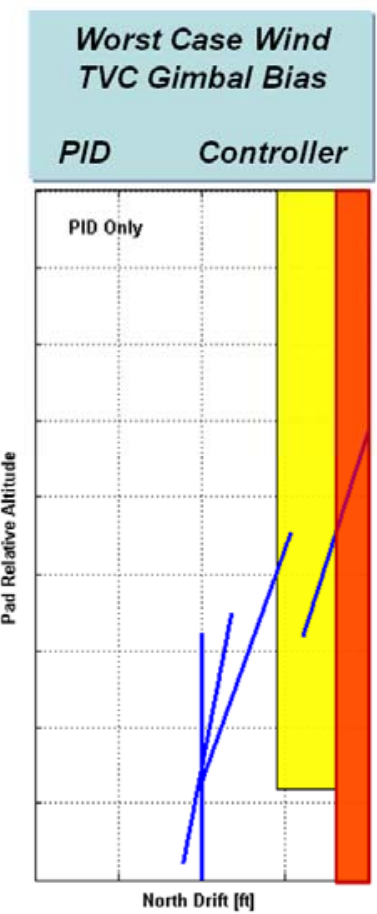

(b)
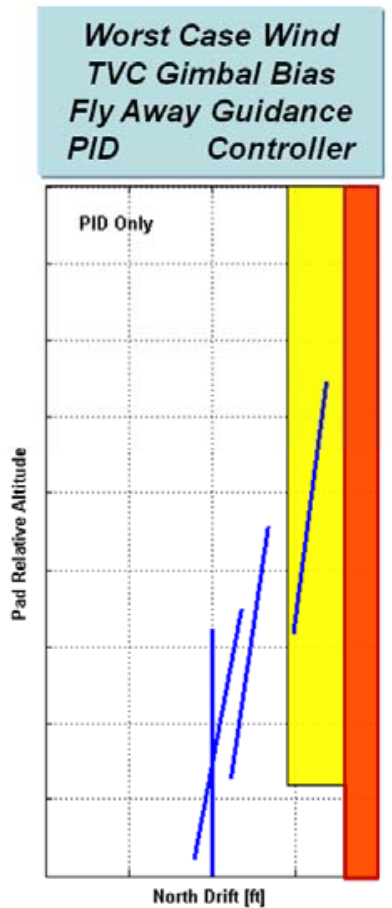

(c)

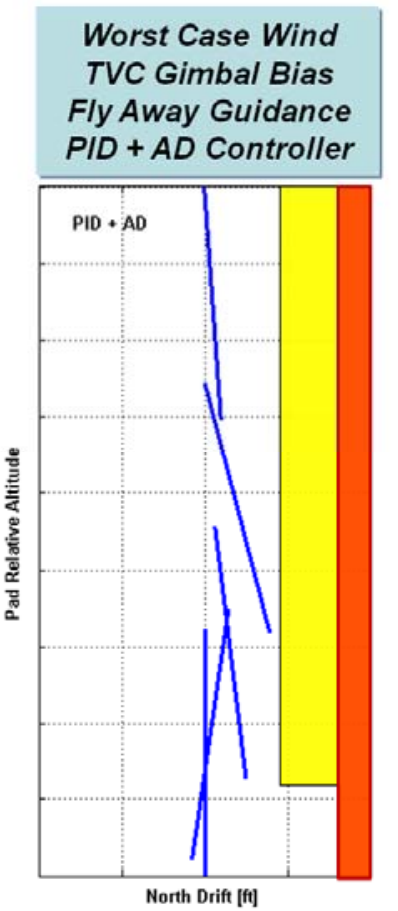

(d)

Figure 6. Tower Clearance Example

During atmospheric flight, the dynamic pressure continuously increases after liftoff as the launch vehicle speeds up before it reaches the peak. The dynamic load is defined as the product of dynamic pressure and angle-of-attack (and/or side-slip-angle). To reduce the dynamic load, it often requires trajectory reshaping with a more accurate wind model (2-3 hours old vs. a monthly average) such that the designed trajectory provides the desired trim angleof-attack to balance the expected aerodynamic load. Trajectory reshaping can reduce the aerodynamic loads by trimming the angle-of-attack; however, it cannot alleviate the transient loads due to wind shears and gusts. On the other hand, the load relief controller is often used to mitigate the transient aerodynamic load. The combination of reshaping the trajectory and load relief control designs can drastically reduce the aerodynamic load which contributes to the significant weight and cost savings for a launch vehicle.

As shown in Figure 5, the blended attitude control and anti-drift / load-relief controller has three competing design goals with a single control variable, i.e., the control design aims to use a single gimbal command to simultaneously achieve attitude control, force balance and moment balance tasks. To effectively reduce the aerodynamic load, the vehicle will be turned into the wind which results in a small amount of attitude tracking error. In Figure 7, two flight control designs are demonstrated: attitude control vs. blended attitude and load-relief control. In Figure 7a, the blended control algorithm effectively reduces the dynamic load (||[Q-alpha Q-beta]||) in comparison with the non-blended attitude control system near the Max Q region. However, the blended control system demonstrates larger attitude errors as shown in Figure 7b. In order to mitigate the built-up trajectory errors for the blended attitude and load-relief control system, load relief control will be designed to gradually phase out once the launch vehicle passes the Max Q region. 


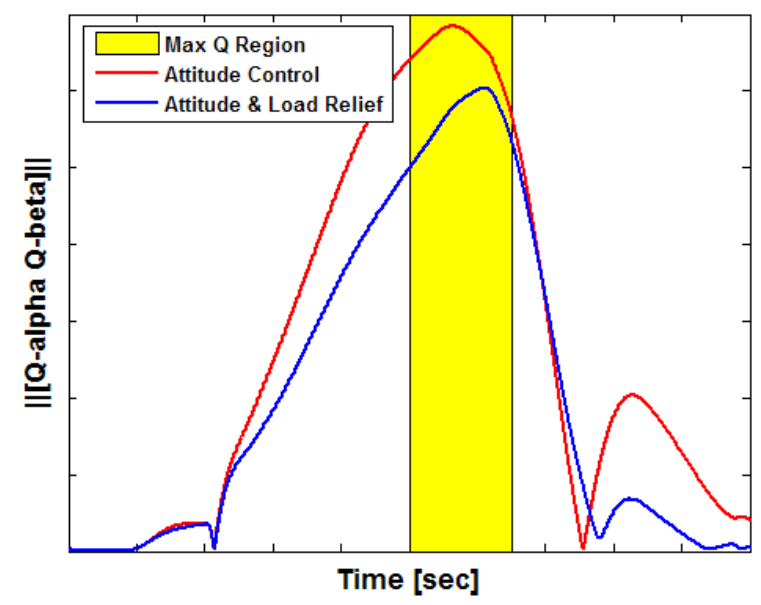

(a)

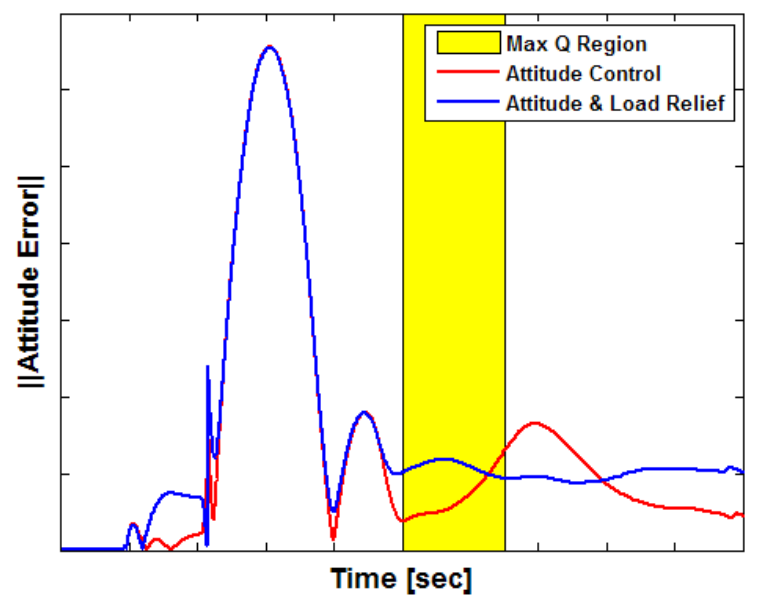

(b)

Figure 7. Load Relief Example

\section{$\underline{\text { Roll Control System }}$}

For a single gimbaled engine launch vehicle like the Ares I, the ascent flight control system uses phase plane autopilots for roll attitude control during powered phases and 3-axis roll, pitch, and yaw rate control during the post Main Engine Cut Off (MECO) coast phase of flight ${ }^{1}$. The roll, pitch, and yaw designs are identical, but use different data loads depending on the axis being controlled and the flight phase. Each phase plane autopilot produces a single command that takes on three states; 0 for drift, +1 for positive rotation and -1 for negative rotation. This command is sent to a jet selection logic that sets the appropriate fixed jet command(s) to "on" or "off."

Because high-frequency flex dynamics have the ability to make the system unstable, it is critical that a low pass filter be employed to attenuate high-frequency flex modes. The rigid and flex plant dynamics are integrated with the flex filter and phase plane controller to create the roll dynamics and control system. The block diagram in Figure 8 outlines the dynamics and control components.

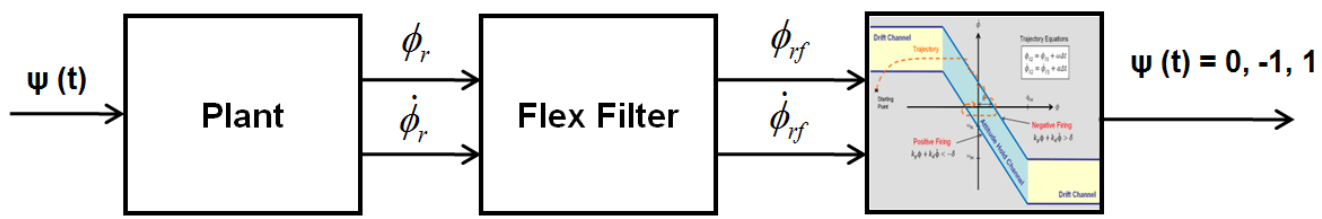

Figure 8. Ares-I Roll Control System Model

The flex filter block consists of a set of low-pass filters to attenuate high-frequency noise while at the same time allowing low-frequency dynamics to feedback into the controller. A phase plane control system regulates attitude tracking and performance. Unlike the PID controller in the TVC control system, the phase plane controller is an inherently nonlinear system which necessitates the use of nonlinear techniques in order to predict the system's behavior. The detail design and analysis of the fixed-jets roll control system can be found in Reference 18-21.

Some launch vehicles have multiple engines which can be gimbaled in pitch and yaw directions. For example, in space shuttle propulsion system, there are two TVC engines on the Solid Rocket Boost (SRB) and three main TVC engines on the Orbiter. A mixing logic will be designed to convert the roll, pitch, and yaw output commands from the flight control system to gimbaled commands which drive the thruster vector. The mixing logic in general can be reconfigured during flight to avoid control system instability due to engine failures. For the multiple gimbaled engines configuration, roll attitude is controlled via differential vectoring. 


\section{Rate Sensor Blending Selection Algorithm}

The design objective of the flight control system is to make the rigid signal follow the command signal. For the flexible launch vehicle, the sensed signals consist of rigid, slosh and flex components. One of the flight control system design goals is to mitigate flex components in the sensed signals before they are sent to the controller. There are two methods to achieve this goal: flex filtering and rate blending. The former method involving bending and torsional filter designs will be elaborated on more in the next section. In this section, the rate blending mechanism will be detailed.

Multiple rate sensors along the structure allow for the blending of the sensed rotational rate, and hence active removal of flex dynamics in the rate channel ${ }^{14}$. In the Space Shuttle sensor package for example, the rate measurement is available from Inertial Measuring Units (IMU) in the forward avionics bay, core body rate gyros (aft mounted) and two SRB rate gyros in the forward compartment. In Ares I, three rate measurements are available: the Inertial Navigation Unit (INU), the inter-stage rate gyro, and the first stage rate gyro as shown in Figure 9. The loop gains of the first and second flex modes are significantly decreased when using the inter-stage rate gyro, since the structural environment is significantly decreased at that location. This point is further illustrated in Figure 9, where it is noted for this flex model and flight time that the inter-stage rate gyro is located near a second mode anti-node, hence is less influenced by the second mode flex dynamics.

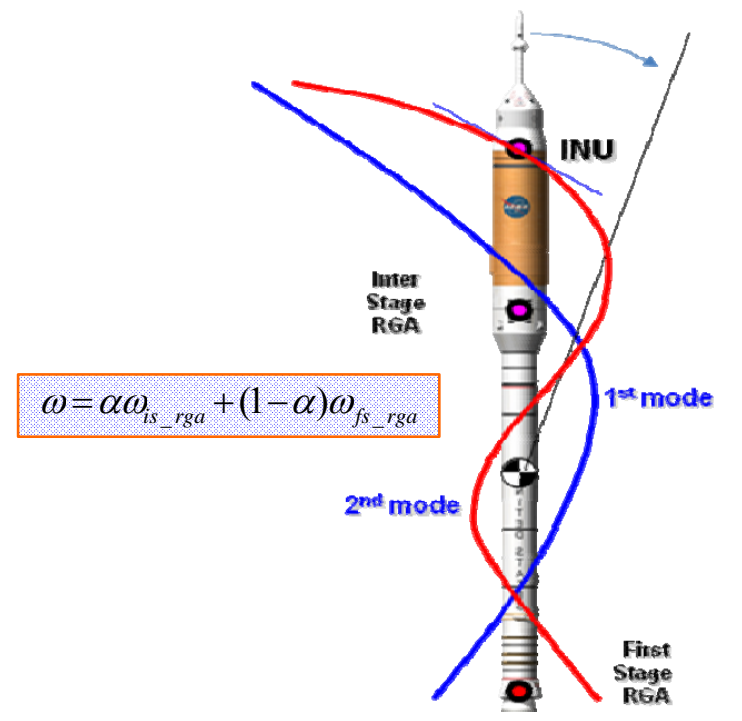

Figure 9. Ares I Rate Sensor Blending

There are two constraints when selecting a sensor blending scheme. First, the blended rate signal must maintain the same phasing as the primary rate signal for the phase stabilized modes. Take the Ares I lunch vehicle as an example; the first flex mode is phase stabilized and the primary rate signal comes from the inter-stage rate gyro. In figure 9, the first bending mode slope at inter-stage rate gyro has an opposite sign from the first bending mode slope at first-stage rate gyro. Therefore, the blending weight must be carefully designed such that the first mode phasing is maintained while the flex amplitude in the blended rate signal is minimized. Second, the rate blending scheme must be robust in the presence of the flex amplitude uncertainty. This implies that the first mode phase cannot be changed for any flex dispersion from either sensor over time.

In Ares I, the sensor weighting is designed such that the inter-stage rate sensors dominate the control law input, hence the first bending mode must be significantly lagged by the filtering to compensate for the sensors being noncollocated with the actuator. Specifically, the inter-stage rate measurement is heavily weighted when blending with the first stage rate gyro. The inter stage rate gyro gain $(\alpha)$ and the first stage rate gyro gain (1- $\alpha)$, are designed to significantly remove second mode flex intrusion, while robustly preserving the phase stabilization on the first flex mode. 


\section{Constrained Optimization Approach for Ares I Flight Control System Design}

It has been previously demonstrated in multiple space applications ${ }^{8-13}$ that bending filters can be designed numerically using a constrained optimization framework. The design parameters are the coefficients of the bending filters. For example, if an $\mathrm{n}^{\text {th }}$ order transfer function architecture is selected for both the attitude and rate filter, the total number of design parameters is $4 \mathrm{n}$.

$$
\begin{aligned}
& F_{\text {attitude }}(s)=\prod_{i=0}^{N-1} \frac{s^{2}+2 x_{4 *_{i+2}} x_{4 *_{i+1}} s+x_{4 *_{i+1}}{ }^{2}}{s^{2}+2 x_{4 *_{i+4}} x_{4 *_{i+3}} s+x_{4 *_{i+3}}{ }^{2}} \\
& F_{\text {rate }}(s)=\prod_{i=0}^{N-1} \frac{s^{2}+2 x_{4 *_{i+2}} x_{4 *_{i+1}} s+x_{4 *_{i+1}}{ }^{2}}{s^{2}+2 x_{4 *_{i+4}} x_{4 *_{i+3}} s+x_{4 *_{i+3}}{ }^{2}}
\end{aligned}
$$

In addition to filter coefficients, PID and AD controller gains/filter coefficients are also included in the design parameters set. A set of feasible parameters must satisfy the following constraints:

(C1). The filter itself must be stable and minimal phase to guarantee stability and performance.

(C2). The bandwidth of the bending filter should be greater than that of the PID controller to avoid rigid performance degradation.

These constraints can be used to set the upper and lower bounds for the bending filter design.

The primary objective of Ares-I control system design is to provide sufficient stability margins in the presence of various parameter uncertainties while maintaining adequate system response. Experience has shown that the following design objectives similar to Reference 14 are adequate:

(O1). Both nominal and perturbed closed-loop Ares-I control systems must be stable.

$(\mathrm{O} 2)$. At least $6 \mathrm{~dB} / 30$ degree rigid gain/phase margin is required for nominal control systems.

(O3). At least $3 \mathrm{~dB} / 20$ degree rigid gain/phase margin is required for perturbed control systems.

(O4). At least $9 \mathrm{~dB}$ nominal gain margin is required for gain stabilized bending modes.

(O5). At least 45 degree nominal phase margin is required for phase stabilized bending modes.

(O6). At least $6 \mathrm{~dB}$ perturbed gain margin is required for gain stabilized bending modes.

(O7). At least 30 degree perturbed phase margin is required for phase stabilized bending modes.

To ensure adequate response to guidance commands, attitude step response specifications are imposed in the optimization problem. The additional constraints minimize the performance degradation due to the addition of the bending filters. These attitude pointing specifications, which include requirements for rise time, percent overshoot, settling time, and steady state errors, vary during the entire flight time.

The Ares-I control systems must also ultimately demonstrate robustness to uncertainties in the plant. The goal is to design bending filters that are robust to uncertainty in structural frequency, mode shape/slope, slosh mass/ damping/frequency, vehicle mass properties, engine mass properties, aerodynamics characteristics and signal transport lag. Once design objectives and constraints are identified, the bending filter design task is ready to be cast as the following constrained optimization problem

$$
\begin{array}{cc}
\min _{x} & f(x) \\
\text { s.t. } & \\
& g(x) \leq 0 \\
& x_{l} \leq x \leq x_{u}
\end{array}
$$

The filter design criteria $(\mathrm{C} 1)$ and $(\mathrm{C} 2)$ can be formulated as inequality constraints. These inequality constraints can be also cast as objectives $f(x)$ in the above multi-objective constrained optimization problem. In general, these objectives are competing with each other. For example, maximizing gain margins usually diminishes phase margins. Therefore, there is no unique solution to this problem. To address this, Pareto optimality ${ }^{22}$ must be applied to 
characterize the objectives. This is accomplished with a weighted sum strategy, which converts the multi-objective problem into a single objective optimization problem.

\section{Design and Analysis of Flight Control Systems Using ASAT}

The design and analysis of the flight control system of a launch vehicle is a complicated task. Mass properties and aerodynamic forces vary considerably over the launch timeline. In addition, propellant slosh varies during upper stage flight as the propellant is consumed. In ASAT, a "frozen time" design and analysis approach, which takes snapshots of the vehicle's time-varying properties at particular flight times, is used. The dynamics equations for a launch vehicle can be classified into three categories: rigid body, bending, and propellant slosh. Rigid body dynamics are those attributable to translation and rotation of the vehicle as a whole. In addition to aerodynamic, thrust, and drag forces, these dynamics include forces due to propellant slosh and the "tail-wags-dog" effect created by adverse torque from gimballing the engine cone.

Configuration and execution of ASAT is controlled through a graphical user interface (GUI) in which users can specify desired flight times, dynamics models, flex filters, flex model variations, sensor locations and weights, and stability analysis settings. Bode, Nichols, and step response plots are generated automatically. Analysis results such as gain and phase margins and overall stability are accessible through the GUI. Most of the system parameters are assigned from the Main GUI, as shown in Figure 10. Some other parameters, such as sensor weighting and controller gains, can be set in their own pop-up GUI.

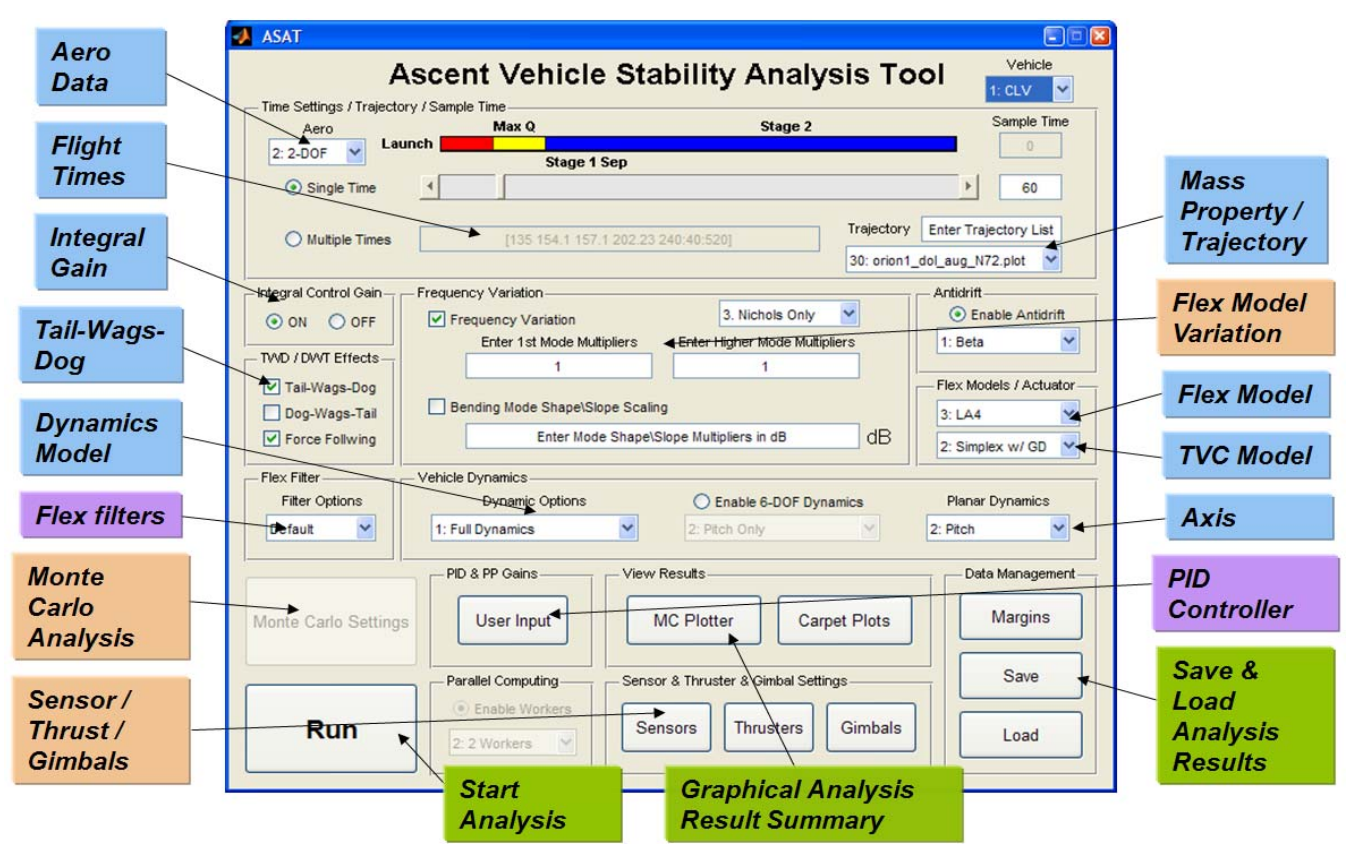

Figure 10. ASAT GUI

To illustrate the design and analysis capability of ASAT, an Ares I flight control systems design example is presented in this section. The Ares I flight control system consists of Thrust Vector Controller (TVC) using identical pitch/yaw autopilot architectures and a phase plane autopilot for roll control during powered flight. During the coast phase after upper stage MECO, independent roll, pitch, and yaw phase plane autopilots are used to command the US Reaction Control System (ReCS) for control. First Stage (FS) and Upper Stage (US) autopilots have the same general structure, but use different data loads such as gains, filters, limit values, and jet selection logic.

The current baseline thrust vector control law is PID with gains and filters scheduled over FS and US flight to accommodate changing flight conditions. The PID gains were designed for rigid body performance, but also to provide as much frequency separation as possible between the rigid body and expected first bending and slosh mode frequencies. For FS, the flex filters were designed to phase stabilize the first bending mode and gain stabilize higher 
bending modes. For US flight, the flex filters gain stabilize all bending modes. Slosh will be damped passively using mechanical baffles in the tanks, and filtering in the control system is used to supply adequate slosh gain/phase margin for gain/phase stabilized slosh modes when passive damping is inadequate for slosh gain stabilization. In FS flight, an AD (Anti-Drift) control law is added in parallel to the PID controller to actively prevent vehicle drifting into the launch tower and reduce aerodynamic load in the maximum dynamic pressure region. The AD gains and filters are also scheduled over FS flight.

The robustness of the Ares I flight control system designs are then be verified in the full Monte Carlo analysis using the Draper Ares Stability Analysis Tool (ASAT) for all dispersed designated trajectories. An ASAT utility imports each nominal datum and identifies the correct flex configuration for each of a series of frozen time points. The analysis time vector is the same for each trajectory and is chosen to provide snapshots of flight with sufficient control authority (thrust magnitude) such that the stability analysis is valid. At each time point along a trajectory, 2,000 open-loop responses in both the pitch and yaw axes are computed after dispersing the relevant parameters according to a pre-generated seed vector. The seeds are consistent among axes and time points along the trajectory. The seed vector is stored and the 2,000 open-loop responses are passed to an automated plotting and margin calculation tool. The margin calculation tool determines the characteristics of the response and tracks both the worstcase margin values and the seed identifiers that created the worst-case systems such that they can be later replicated. The following margins and characteristics are identified if they exist:

Rigid gain margin: minimum gain margin at rigid phase crossover frequencies.

Flex gain margin: negative of maximum flex peak amplitude of gain stabilized modes.

Rigid phase margin: minimum phase margin at rigid gain crossover frequency.

Flex phase margin: minimum phase margin at the flex gain crossover frequencies.

The relevant characteristics are illustrated on the Nichols response shown in Figure 11. The nominal and robust frequency margin requirements for the current design are listed in $(\mathrm{O} 2)$ to $(\mathrm{O} 7)$.

In ASAT Monte Carlo analysis, frequency responses of the nominal and all dispersed control systems are depicted in both Nichols and Bode plots as shown in Figure 12. The requirements of the nominal rigid margins are indicated by the black diamond encompassing the $(0 \mathrm{~dB},-180 \mathrm{deg})$ point in the Nichols chart - the top and bottom vertices denote the rigid gain margins, and the right and left vertices indicate the rigid and flex phase margins, respectively. Similarly, the red diamond shows the rigid margin requirements for the dispersed cases. The nominal and dispersed flex gain margin requirements are drawn as black and red horizontal lines, respectively, in both Nichols and Bode plots. 


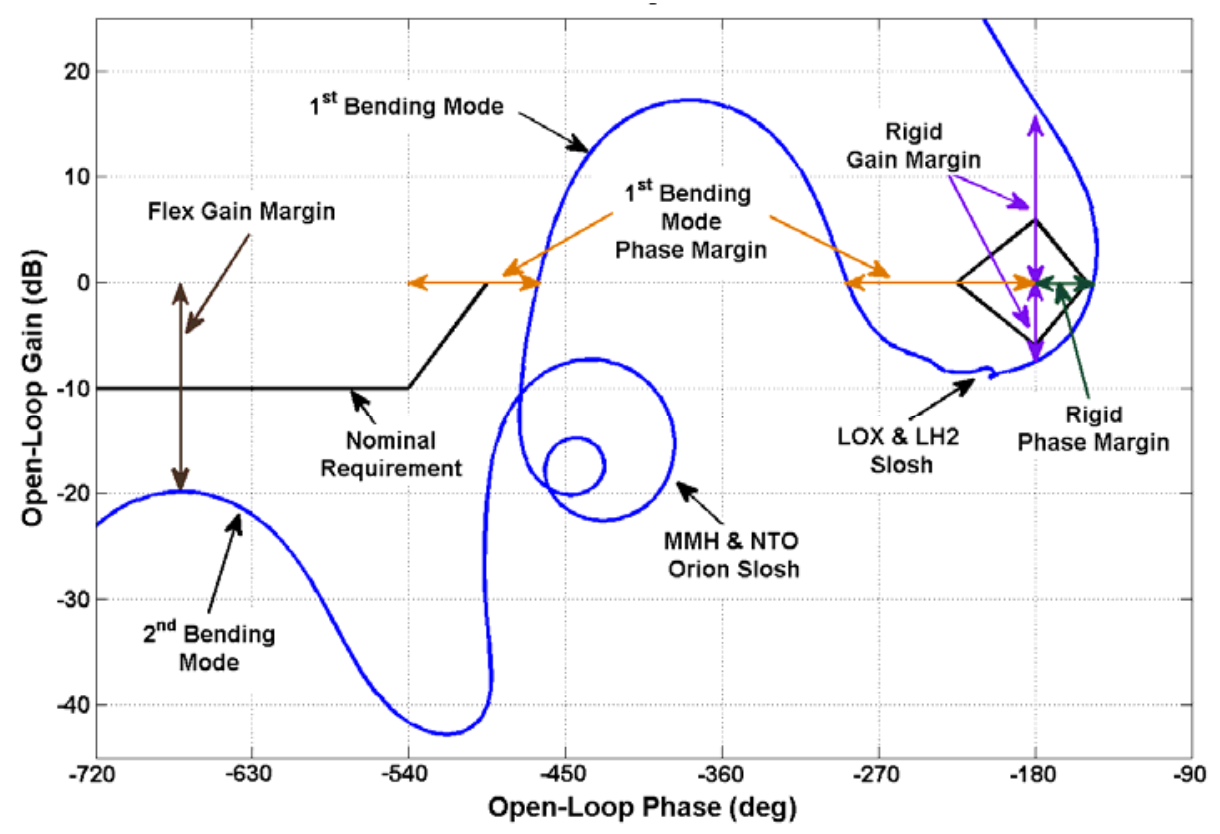

Figure 11. Stability Margin Definition Examples

MC 60sec pitch 2: 1000 Runs Bode Magnitude Plot: Flight Time at $60 \mathrm{sec}$

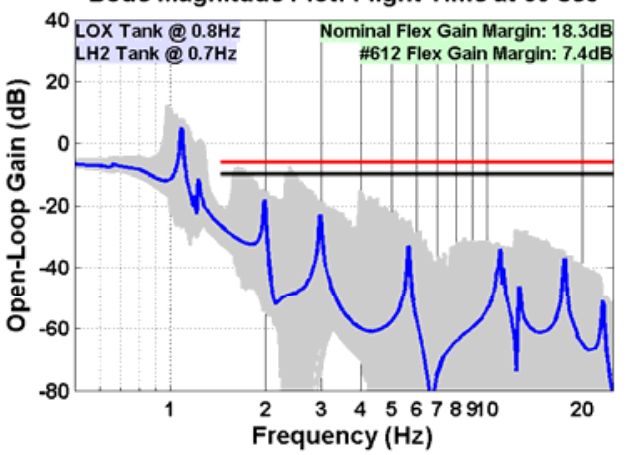

MC 60sec pitch 2: 1000 Runs Bode Phase Plot: Flight Time at $60 \mathrm{sec}$

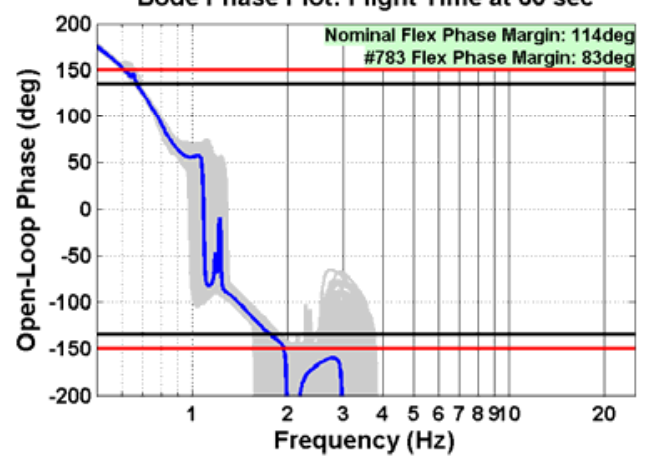

MC 60sec pitch 2: 1000 Runs Nichols Chart: Flight Time at $60 \mathrm{sec}$

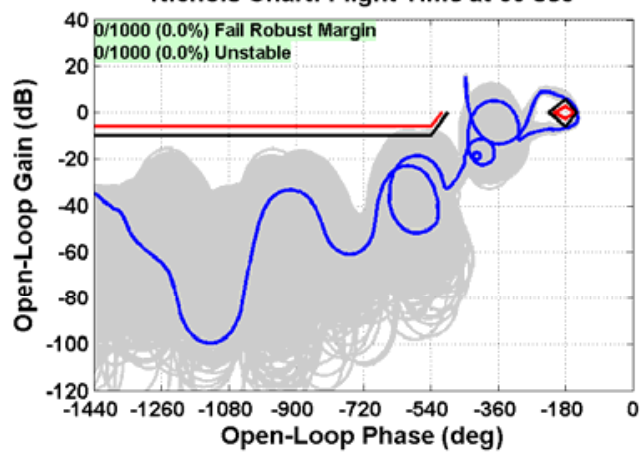

MC 60sec pitch 2: 1000 Runs Nichols Chart: Flight Time at $60 \mathrm{sec}$

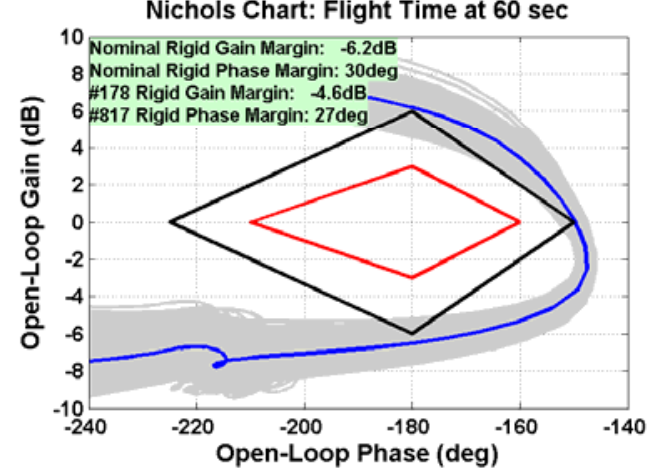

Figure 12. ASAT Monte Carlo analysis example 


\section{Summary}

A launch vehicle represents a unique and challenging flex-body structural environment for control system design. To ease the complication of a launch vehicle flight control system design task, an effective design tool, ASAT, with a great deal of analysis capabilities within a user friendly environment is developed. The flight control system design methodology in ASAT was based on a numerical constrained optimization approach to maximize performance while meeting stability margins requirements. "Tower Clearance" and "Load Relief" designs have been achieved for liftoff and max dynamic pressure flight regions, respectively, in the presence of large wind disturbances. The robustness of the flight control system designs has been verified in the frequency domain Monte Carlo analysis using ASAT.

\section{References}

1 Jang, J., Alaniz, A., Hall, R., Bedrossian, N., Hall, C., Ryan, S., Jackson, M., “Ares I Flight Control System,” AIAA GN\&C Conference, Toronto, Canada, 2010, AIAA-2010-8442.

2 Hall, C., Lee, C., Jackson, M., Whorton, M., West, M., Brandon, J., Hall, R., Jang, J., Bedrossian, N., Compton, J., and Rutherford, C., "Ares I Flight Control System Overview," AIAA GN\&C Conference, Honolulu, HI, 2008, AIAA-2008-6621.

3 Dukeman, G., "Atmospheric Ascent Guidance for Rocket-Powered Launch Vehicles", AIAA Paper 2002-4559, Proceedings of the AIAA Guidance, Navigation, and Control Conference, Monterey, CA, August 5-8, 2002.

4 Edinger, L.D., "The Space Shuttle Ascent Flight Control System," AIAA 76-1942, Guidance and Control Conference, San Diego, CA, Aug. 16-18, 1976. AIAA 1976, pp. 225-235.

5 Oppenheim, A. V, and Schafer, R. W., Digital Signal Processing, Prentice-Hall Company, Inc., Englewood Cliffs, New Jersey, 1975.

6 Friedlander, B., and Porat, B, "The Modified Yule-Walker Method of ARMA Spectral Estimation," IEEE Transactions on Aerospace Electronic Systems, AES-20, No. 2, March 1984, pp. 158-173.

7 Strum, R. D. and Kirk, D. E., First Principles of Discrete Systems and Digital Signal Processing, Addison-Wesley Publishing Company, Inc., New York, April 1989.

8 Jang, J., Hall, R., Bedrossian, N., and Hall, C., "Ares-I Bending Filter Design Using A Constrained Optimization Approach," AIAA GN\&C Conference, Honolulu, HI, 2008.

9 Jang, J., Bedrossian, N., Lee, A, Spanos, P., “A Constrained Optimization Approach for CMG Robust Flex Filter Design”, AIAA GN\&C Conference, August 2002.

10 Jang, J., Lee, A., Bedrossian, N., Spanos, P., "Design of Robust Nash Game Theoretic Controllers with Time Domain Constraints," American Control Conference, 2003.

11 Bedrossian, N., Jang, J., Alaniz, A., Johnson, M., Sebelius, K., Mesfin, Y., "International Space Station US GN\&C Attitude Hold Controller Design for Orbiter Repair Maneuver," AIAA GN\&C Conference, August, 2005.

12 Postma, B., Jang, J., Bedrossian, N., Spanos, P., "Robust Constrained Optimization Approach for International Space Station Centrifuge Rotor Auto-Balancing Controller," AIAA GN\&C Conference, August, 2005.

13 Jang, J., Bedrossian, N., Hall, R., Norris, L. H., Hall, C., Jackson, M., "Initial Ares-I Bending Filter Design," $30^{\text {th }}$ AAS Guidance and Control Conference, AAS 07-078, Breckenridge, Colorado, February 2007.

14 Garner, D., "Control Theory Handbook," NASA Marshall Space Flight Center, NASA TM X-53036, April 22, 1964.

15 Frosch, J.A., Vallely, D.P., "Saturn AS-501/S-IC Flight Control System Design”, Journal of Spacecraft, Vol. 4, No. 8, August 1967.

16 Haussermann, W., Duncan, R.C, "Status of Guidance and Control Methods, Instrumentation, and Techniques as Applied to the Apollo Project", Agardograph 92, NASA Code ATSS-AD, October 1964.

17 Greensite, A. L., Analysis and Design of Space Vehicle Flight Control Systems, Spartan Book, New York, 1970.

18 Penchuk, A., Hattis, P., and Kubiak, E., “A Frequency Domain Stability Analysis of a Phase Plane Control System,” Journal of Guidance, Vol. 8, No. 1, 1984

19 Wertz, J., Spacecraft Attitude Determination and Control, Boston, MS: D. Reidel Publishing Company, 1995.

20 Gelb, A., and Velde, W. V., Multiple-Input Describing Functions and Nonlinear System Design, New York, NY: McGrawHill Book Co., 1968.

21 Jang, J., Plummer, M., Bedrossian, N., Hall, C., Jackson, M., and Spanos, P., “Absolute Stability Analysis of a Phase Plane Controlled Spacecraft," 20th AAS/AIAA Space Flight Mechanics Meeting, AAS 10-112, San Diego, CA, February 2010.

22 Rao, S. S., "Game Theory Approach for Multi-objective Structural Optimization," Computers \& Structures, Vol. 24, No. 1, 1987, pp. 119-127. 